\title{
Psychometric Characteristics of the ECR-RS, Structure of the Relationship between Global and Specific Attachment - Cross-Validation of the Results Using the Czech and Original Versions of the ECR-RS
}

\author{
Aneta Siroňová $^{1}$ (D) Hynek Cígler $^{1,2}$ (iD) Stanislav Ježek ${ }^{1,2}$ (iD) Lenka Lacinová ${ }^{1,2}$ \\ ${ }^{1}$ Department of Psychology, Faculty of Social Studies, Masaryk University, Joštova 10, Brno, Czech Republic \\ ${ }^{2}$ The Institute for Research on Children, Youth and Family, Faculty of Social Studies, Masaryk University, Joštova 10, \\ Brno, Czech Republic
}

\begin{abstract}
Several tools to measure attachment style in adulthood have been developed in the past three decades; however, their dimensionality is still unclear, with the main unanswered question being that of global attachment. In the Relationship Structures Questionnaire, ECR RS (Fraley et al., 2011), respondents rate their relationship to four attachment figures (mother, father, close friend and partner). The paper assesses its dimensionality to test the structure of global attachment. We used a Czech sample $(N=1023)$ and an international sample (Hudson et al., 2015; $N=1095$ ) to compare a hierarchical model, in which the figure specific attachment factors are partially the product of global attachment, with a bifactor model, in which the global attachment factors directly affect the responses in questionnaires. The bifactor model fits the data better and it lends support to the hypothesis that global attachment relates to human behavior directly and is not mediated by specific attachment to different figures. The limitations of this finding are discussed.
\end{abstract}

Key words: attachment, global attachment, specific attachment, ECR-RS, bifactor model, structure of attachment

In the past three decades, attachment theory has become a major perspective on close interpersonal relationships in adulthood. Many measures have been developed to assess adult attachment. In addition to the category classifications of attachment styles based on a developmental perspective that originated in Ainsworth, Blehar, Waters, and Wall's (1978) research, a dimensional approach, using two latent dimensions, is applied in measuring

Correspondence concerning this article should be addressed to Aneta Siroňová, Department of Psychology, Faculty of Social Studies, Masaryk University, Joštova 10, Brno, Czech Republic.

E-mail: cvrckova.aneta@gmail.com

The Online Supplement to this article can be accessed at https://journals.savba.sk/index.php/studiapsychologica/article/view/111/41

Received October 18, 2019 
adult attachment (Griffin \& Bartholomew, 1994). The continuous distribution of individual differences in attachment has been highly supported by the results of taxometric analysis (i.e., Fraley, Hudson, Heffernan, \& Segal, 2015; Fraley \& Roisman, 2014).

Attachment measurement tools differ in their level of specificity. Some self-report questionnaires focus on "global attachment", i.e., how people feel in general in their relationship; others consider the type of relationship, e.g., romantic relationships. The variability of attachment through different relationship contexts has been empirically supported (e.g., Baldwin et al., 1996). One simulation study supported the existence of both global attachment as well as relationship-specific attachment (Fraley, 2002), but to this date, the relationship between global and specific attachment has not been satisfactorily evaluated.

\section{The Experiences in Close Relationships - Relationship Structures Questionnaire}

Currently, the most widely used method for assessing attachment styles is the self-report questionnaire, especially the various versions of the Experience in Close Relationships (ECR) (Crowell, Fraley, \& Roisman, 2016). One of the most recent versions is the Experiences in Close Relationships - Relationship Structures Questionnaire (ECR-RS) (Fraley, Heffernan, Vicary, \& Brumbaugh, 2011). The ECR-RS has its origins in the ECR (Brennan, Clark, \& Shaver, 1998) and the Experience in Close Relationship - Revised (ECR-R) developed by Fraley, Waller, and Brennan (2000). The ECR-RS captures the two basic dimensions of attachment: anxiety and avoidance. Attachment anxiety reflects the extent to which people tend to worry about the availability and responsiveness of the attachment figure; attachment avoidance reflects the extent to which people are uncomfortable depending on others and opening up to them (Fraley et al., 2011).

The ECR-RS has been designed to assess individual differences in attachment anxiety and attachment avoidance within and across a variety of relational contexts (e.g., mother, father, romantic partner, best friend). The method has been developed to overcome several methodological limitations of previous self-report measures - ambiguity of what kind of relationship is being assessed or (on the contrary) a too narrow focus of methods (e.g., ECR assesses romantic attachment exclusively). Moreover, previous measures of attachment also did not allow the study of within-person variation of attachment avoidance and anxiety in different relational contexts (Fraley et al., 2011). The authors also suggested that the ECR-RS could be used to assess global attachment anxiety and global attachment avoidance, which could be represented as a linear combination (mean) of anxiety/avoidance across the four examined relationships. Unfortunately, this assumption has not been tested.

Despite the valuable contribution of the ECR-RS to the field of attachment research, some psychometric characteristics of the ECR-RS still remain unclear. First, Fraley et al. (2011) used exploratory Factor Analysis with varimax rotation, which presumes an orthogonality of factors. However, the correlation between attachment anxiety and avoidance was supported by the meta-analysis study (Cameron, Finnegan, \& Morry, 2012). Second, studies using factor analysis detected cross-loadings of the two-last avoidance scale items with the anxiety scale (Feddern Donbaek \& Elklit, 2014), and some residual correlations had to be allowed for an adequate fit of the model to the data (Moreira, Martins, Gouveia, \& Canavarro, 2015). Third, existing studies have thus far not examined the factor invariance across gender. It is possible that dif- 
ferent interpretations and response tendencies could occur depending on gender when evaluating feelings and behavior in close relationship. Women increasingly participate in research concerned with relationships (e.g., Del Giudice, 2011) and an implicit tendency to compare ourselves with the typical behavior of the same gender was described (e.g., Biernat, 2003). Measurement invariance across gender has so far only been supported in the ECR-R (Favez, Tissot, Ghisletta, Golay, \& Cairo Notari, 2016). Fourth, Fraley et al. (2011) also did not empirically test assumed global avoidance and global anxiety.

\section{Global versus Specific Attachment}

Fraley et al. (2011) shifted the research focus away from global attachment (how a person feels in all relationships in general) or from a specific type of relationship (i.e., how a person feels in a romantic relationship) when they included the four specific relationship contexts in the measurement of attachment. Collins and Read (1994) proposed theoretical models of a hierarchical structure of specific and global representations of attachment. Overall, Fletcher, and Friesen (2003) tested three hypothetical models (see Figure 1). The third model fitted the data best in comparison to the first and the second models. Both global and specific attachment were supported. The relationship between attachment avoidance and attachment anxiety is stronger in more similar interpersonal contexts (mother - father) than in different social categories (mother - romantic partner). Their results contributed to the explanation of the variabil-
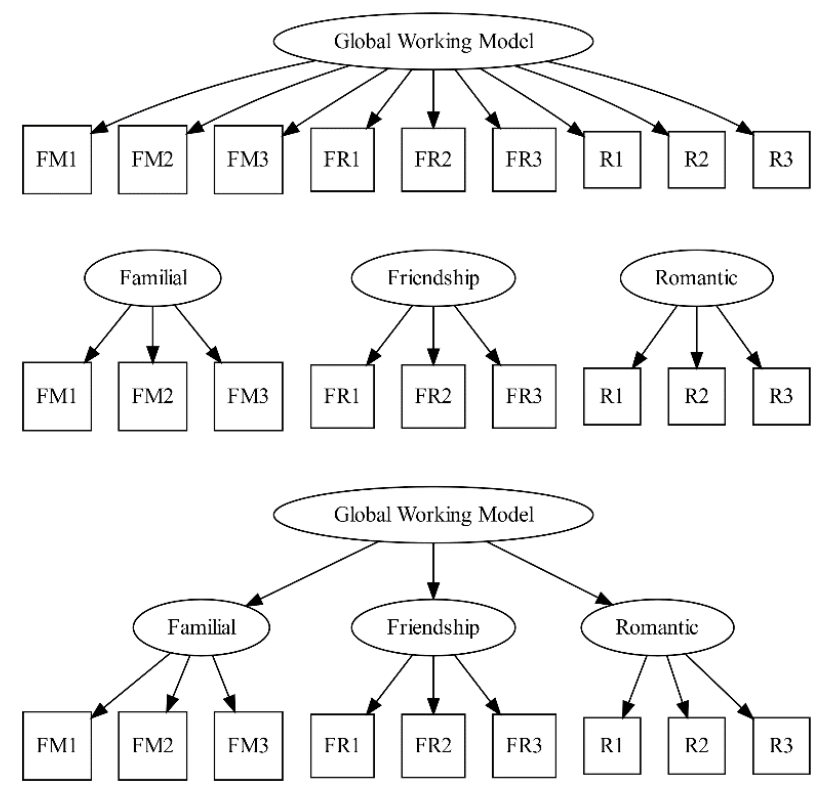

Figure 1 Hypothetical models of global and specific attachment - models for Confirmatory factor analysis; $F M=$ family observed variables, $F R=$ friendship observed variables, $R$ = romantic observed variables (Overall et al., 2003). 
ity of attachment across specific relationship domains (i.e., mother, partner). The difference between attachment relationships has been supported by other research through Hierarchical Linear Modeling analysis (Sibley \& Overall, 2008).

Fraley (2007) proposed a connectionist perspective as an alternative framework for understanding the structure of global and specific attachment. According to Fraley (2007), a person can develop representations of specific experiences in different relationships and the shared core of these creates the global representation. Therefore, global representations of attachment may develop based on different relationship experiences, independent of the particular social categories.

By constructing the ECR-RS, Fraley et al. (2011) made it possible to compare a person's attachment to different attachment figures meaningfully. Their results suggest that there is relatively high variability in intrapersonal attachment avoidance and anxiety. Moreira et al.'s (2015) study, on the other hand, showed that correlations were strong across all four attachment contexts and that there was greater consistency of attachment anxiety across different relationships, which could suggest the existence of global attachment anxiety.

To the best of our knowledge, no study has yet empirically assessed the global factors measured by the ECR-RS. The above-mentioned Overall et al.'s study (2003) also has several methodological shortcomings. The authors measured attachment only in "general relationships" (using the Adult Attachment Questionnaire) and applied the categorical model of attachment, but as was previously mentioned, a dimensional approach appears more preferable. Next, the authors did not estimate the reliability of the second-order factor in the third model (see Figure 1). Considering its structure, the ECR-RS is a suitable measure for the clarification of the relationship between specific and global attachment anxiety and avoidance.

This paper consists of three studies. The purpose of Study 1 was a) to validate the Czech version of the ECR-RS and thus confirm it has the same factor structure as the original ECR-RS (see Figure 2); b) to assess the factor invariance across genders; c) to test the plausibility of estimating global attachment anxiety and global attachment avoidance and compare the relationship between possible global factors and specific factors using a hierarchical structural model and a bifactor model (see Figure 3).

By comparing a hierarchical and a bifactor model, the hypotheses about indirect/mediated effect (a higher-order model) and direct effect of global factors (a bifactor model) are compared. The hierarchical model, in the

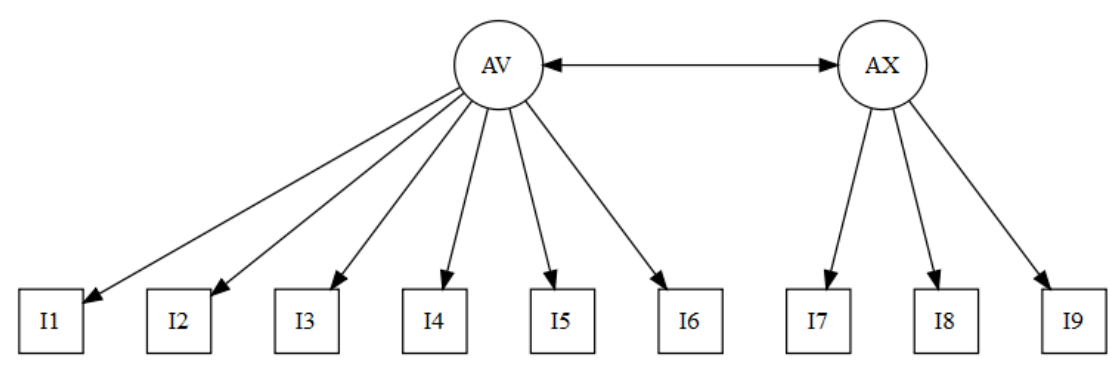

Figure 2 Original models of the ECR-RS; $A V=$ attachment avoidance, $A X=$ attachment anxiety. 

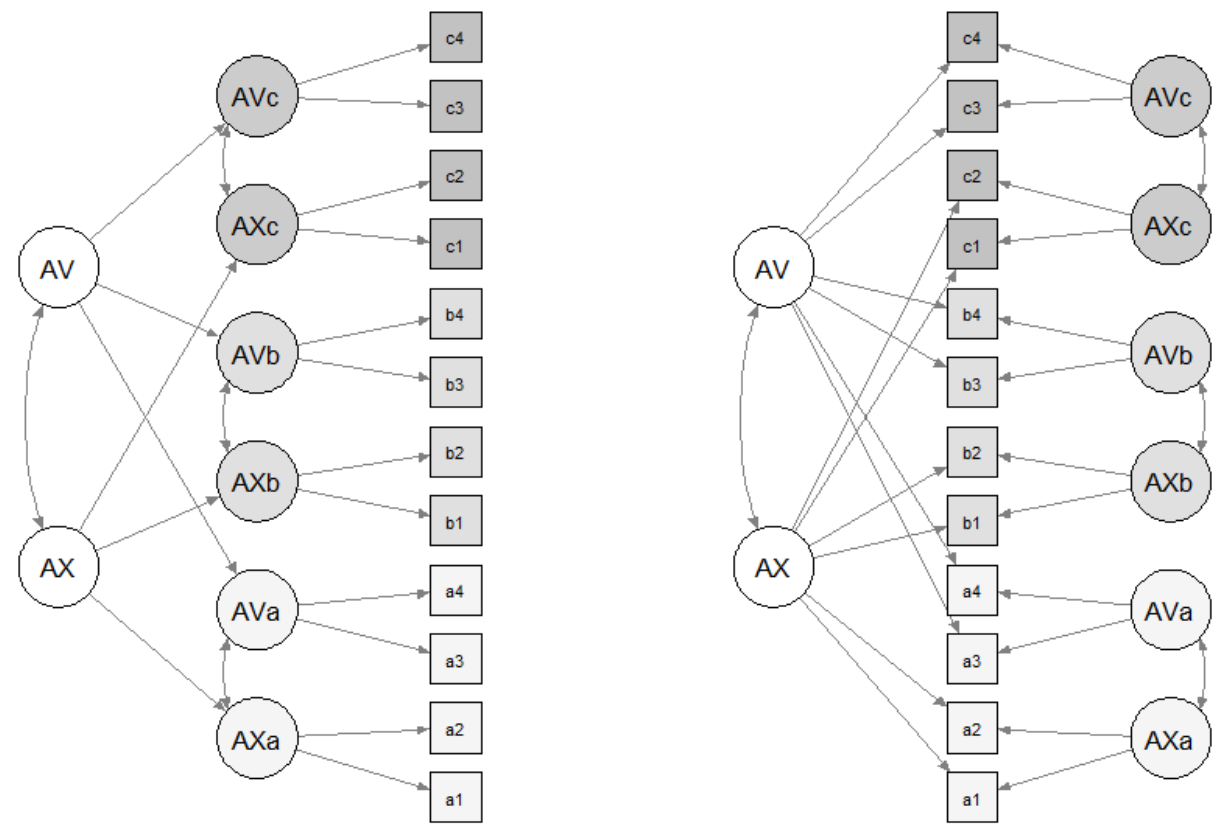

Figure 3 Visual illustration of the difference between higher-order model (left) and bifactor model (right); the most important difference is in the direction of regression arrows - in the higher-order model (left), regression arrows direct to the first-order factors; in the bifactor model (right), regression arrows direct to items. The number of both items and attachment figures were reduced in this illustration to make the graph clearer. $A V=$ avoidance, $A X=$ anxiety; a, b, c-symbolic label for three different attachment figures; a1-c4-items.

context of attachment, presumes that global attachment avoidance (or global anxiety) affects avoidance (or anxiety) in a relationship with particular attachment figures. The bifactor model suggests that global attachment avoidance and global attachment anxiety directly affects our experience in a relationship, together with specific attachment avoidance and attachment anxiety toward specific persons (attachment figures). This type of bifactor model with more general factors (in our case with two) are sometimes labeled as "two-tier models" (Cai, 2010). Global and specific attachment factors are orthogonal in the bifactor model and thus the specific (in bifactor) and first-order (in hierarchical) model are interpreted and conceptualized differently. The specific factor in the hierarchical model can be understood as a "part" of the global factor. The specific factor in the bifactor model can be understood as the specific variance of items, which has nothing in common with the global factor. The study of Overall and colleagues (2003) provides some evidence to support a hierarchical structure of attachment. But nonetheless, as Gillath, Karantzas, and Fraley (2016) stated, other plausible organizational structures 
of adult attachment were not tested to determine whether the hierarchical model does indeed represent the most appropriate structure of attachment representations. The study of Klohnen, Weller, Luo, and Choe (2005) supported the distinctiveness of global and specific attachment and highlighted different predictive validity of global and specific attachment representations for life outcomes. The advantage of the bifactor model is the possibility to compare the degree to which our specific and global attachment relates to our experience in a relationship. A bifactor model is attractive theoretically because it allows us to conceptually separate an individual's characteristics (global factors) from the characteristics of their partners, who are not passive receivers of the individual's attachment. For example, to agree with an anxiety item one can be anxious but additionally one can have an anxiety provoking partner.

The purpose of Study 2 was to replicate the findings from the Czech sample on data collected using the original English version of ECR-RS.

The purpose of Study 3 was to compare the results of Study 1 and Study 2 (Czech and English versions of ECR-RS).

We performed these analyses in the same order as we described them here, which means that we used the knowledge of factor structure in the Czech sample (from Study 1) and we constructed and cross-validated the same model in the original population (Study 2). Then both samples were compared (Study 3).

Besides assessing the psychometric properties of the Czech and the English ECR-RS, this study has the potential to contribute to the attachment literature about the possible types of association between specific attachment and global attachment. To the best of our knowledge, no study has considered this using ECR-RS (as the only attachment questionnaire validated for measuring of more attachment figures), and structural equation modeling.

\section{Study 1 \\ Method}

\section{Participants}

Data from 1,023 Czech participants who reported about all four relationship figures in the ECR-RS were used in this study (participants who did not respond to one or more domains were excluded because of our lack of knowledge about their motivation not to answer the specific domain). The sample was recruited using social network sites aimed at young adults mainly in the South Moravia region. Their age ranged from 16 to 30 years $(M=21.57, S D=$ 1.58). The sample comprised mainly women $(78 \%, n=796)$. Most participants reported being in dating or marital relationships (56\%, $n=574)$ with the average length of their relationship being 29 months $(S D=22.6)$, and most did not agree that "it would be better to try to find a different partner" (84\%, $n=482$ ), only $2 \%$ agreed completely $(n=12)$. The majority of participants had parents still living together (61\%, $n=626)$; 255 respondents reported a break-up of their parents $(25 \%)$, and the parents of 5 participants $(0.5 \%)$ had never lived together. Most participants lived with both biological parents $(92 \%, n=938)$, only $4(0.4 \%)$ lived with adoptive or foster parents.

\section{Materials and Procedure}

The Experience in Close Relationships-Relationship Structures (Fraley et al., 2011) was designed to assess attachment orientation to four attachment figures - mother, father, romantic partner, and best friend. The same nine items are used for each attachment figure (36 in total), six items measure attachment avoidance and three items attachment anxiety. For each item, participants were 
asked to indicate on a 7-point scale the extent to which they agree or disagree with the item ( 1 = strongly disagree; 7 = strongly agree). The first four items are reverse keyed. Participants could indicate the type of romantic partner they were referring to - "current partner", "ex-partner", and "partner they imagine and wish". Cronbach's alpha reliability estimates in our sample were the following: "mother domain" $\alpha_{\text {avoid }}=0.92, \alpha_{\text {anx }}=0.74 ;$ "father domain" $\alpha_{\text {avoid }}=0.88, \alpha_{\text {anx }}=0.81$; "partner domain" $\alpha_{\text {avoid }}=0.88, \alpha_{\text {anx }}=0.87$; "best friend domain" $\alpha_{\text {avoid }}=0.89, \alpha_{\text {anx }}=0.88$.

The data from the five-year longitudinal Czech project Paths to Adulthood were used in this study. The project focused on psychosocial development in emerging adulthood. Data collection started in autumn 2012. The ECR-RS was administered in September 2013 along with a fixed battery of other measures of close relationships. First, general questions about close relationships were asked, second, the ECR-RS was administered. Participants responded to the items in an online questionnaire (Macek et al., 2016). The ECR-RS was independently translated into Czech by two translators. Both versions were then translated back into English by a third person, who was not familiar with the original questionnaire. Differences were used to finalize the Czech version.

\section{Data Analysis}

The open-source program R ( $R$ Core Team, 2016) was used for analyses. Some options of the Likert Scale had a very low response rate and seemed to be redundant - respondents were not able to appropriately respond using the 7-point response scale. In some subsamples used for further analyses (i.e., invariance testing), some response categories were completely missing, which would have complicated these analyses. The scale was thus short- ened to a 4-point scale for all the following analyses (collapsing answers with the values 2 and $3 ; 4$ and 5; 6 and 7). This should not bias the results, as we used categorical factor analysis and items were considered to be ordinal, not continuous (see below).

In confirmatory factor analysis (CFA), indicators were modeled as ordinal and the Diagonally Weighted Least Squares (DWLS) estimator of polychoric correlation matrix with robust correction (WLSMV) was used. Missing data were treated using pair-wise deletion. CFA analyses were conducted using the packages "lavaan" version 0.5-23.1097 (Rossel, 2012), "semTools" version 0.4-14 (semTools Contributors, 2016) and "semPlot" version 1.1 (Epskamp, 2014).

The CFA models were respecified based on modification indices and the inspection of residual correlation matrices in Study 1 and these results were crossvalidated with a different sample in subsequent Study 2 .

Model comparisons, especially in invariance tests, were performed using Satorra and Bentler's (2010) procedure, supplemented by difference fit indices $\triangle T L I, \triangle R M S E A$ and $\triangle S R M R$, with cutoff values according to Chen (2007) and Sass, Schmitt, and Marsh (2014). Measurement invariance testing approach is described in Online Supplement.

Coefficient omega was used for calculating the reliability estimation of the second-order factors in the hierarchical model, $\omega_{2}$ indicates the proportion of the second-order factor explaining the variance at first-order factor level, $\omega_{p}$ indicates the proportion of observed variance explained by the second-order factor after partialing the uniqueness from the first-order factor. For the bifactor model, values of coefficient omega indicate the specific value of one latent factor with the removed influence of other factors.

All the data and research scripts are available online at https://osf.io/dz83k/?view on-

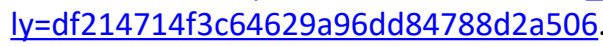




\section{Results}

Confirmatory Factor Analysis for each Relational Domain - Czech sample

As the first step, separate CFAs were conducted for each relational domain. The whole sample ( $N=1023$ ) was used for the mother, father, and best friend domains. For the partner domain, only those participants who responded about their current romantic partner $(N=581)$ were selected. The original structure of the two-factor model does not fit the data well $\left(\chi^{2}=600.060, d f=26, p<.001\right.$, $C F I=0.973, R M S E A=0.147$ with $C l_{90 \%}[.137$, $.157], S R M R=.075)$. A respecification of the models had to be conducted [for answers to all relational domains: correlation of residuals of item 5 and item 6 and cross-loading for item 7 is allowed; for answers only to mother: the parameter of item 9 is constrained to be equal to item 7 (load on attachment anxiety)]. The fit indices for the original and respecified models and standardized parameters are available in Tables 1 and 2 .

Table 1 Fit indices for original and respecified 2-factor models for each relational domain Czech sample

\begin{tabular}{|c|c|c|c|c|c|c|}
\hline Model & $\chi^{2}$ & $d f$ & CFI & $T L I$ & RMSEA $\left[\mathrm{Cl}_{90 \%}\right]$ & SRMR \\
\hline \multicolumn{7}{|c|}{ ECR-RS answers to mother $(N=1023)$} \\
\hline original model & $600.060^{* * *}$ & 26 & 0.973 & 0.963 & $\begin{array}{c}0.147 \\
{[0.137,0.157]}\end{array}$ & 0.075 \\
\hline respecified model & $371.418^{* * *}$ & 25 & 0.984 & 0.977 & $\begin{array}{c}0.116 \\
{[0.106,0.127]}\end{array}$ & 0.063 \\
\hline \multicolumn{7}{|c|}{ ECR-RS answers to father $(N=1023)$} \\
\hline original model & $936.881^{* * *}$ & 26 & 0.946 & 0.925 & $\begin{array}{c}0.185 \\
{[0.175,0.195]}\end{array}$ & 0.095 \\
\hline respecified model & $278.274^{* * *}$ & 24 & 0.985 & 0.977 & $\begin{array}{c}0.102 \\
{[0.091,0.113]}\end{array}$ & 0.056 \\
\hline \multicolumn{7}{|c|}{ ECR-RS answers to actual romantic partner $(N=581)$} \\
\hline original model & $325.926^{* * *}$ & 26 & 0.960 & 0.945 & $\begin{array}{c}0.141 \\
{[0.128,0.155]}\end{array}$ & 0.086 \\
\hline respecified model & $83.780^{* * *}$ & 24 & 0.992 & 0.988 & $\begin{array}{c}0.066 \\
{[0.051,0.081]}\end{array}$ & 0.041 \\
\hline \multicolumn{7}{|c|}{$\underline{E C R-R S \text { answers to best friend }(N=1023)}$} \\
\hline original model & $716.019^{* * *}$ & 26 & 0.966 & 0.953 & $\begin{array}{c}0.161 \\
{[0.151,0.171]}\end{array}$ & 0.083 \\
\hline respecified model & $315.901^{* * *}$ & 24 & 0.986 & 0.978 & $\begin{array}{c}0.109 \\
{[0.099,0.120]}\end{array}$ & 0.059 \\
\hline
\end{tabular}

Note. ${ }^{* * *} p<.001$. Originally proposed model: items 1-6 measure attachment avoidance, items 7-9 attachment anxiety. Respecified model: residual covariance between items 5 and 6 was allowed; item 7 was cross-loaded on both factor; and loadings of items 7 and 9 were constrained to the same value due to convergence problems and negative residual variances. 


\begin{tabular}{|c|c|c|c|c|c|c|c|c|}
\hline Item & AVM & AXM & AVF & AXF & AVP & AXP & AVBF & AXBF \\
\hline 1 & .84 & & .82 & & .85 & & .83 & \\
\hline 2 & .97 & & .94 & & .96 & & .96 & \\
\hline 3 & .93 & & .94 & & .92 & & .91 & \\
\hline 4 & .73 & & .76 & & .72 & & .75 & \\
\hline 5 & .79 & & .61 & & .64 & & .68 & \\
\hline 6 & .73 & & .62 & & .68 & & .70 & \\
\hline 7 & .12 & .90 & .31 & .72 & .32 & .67 & .28 & .77 \\
\hline 8 & & .67 & & .79 & & .79 & & .89 \\
\hline \multirow[t]{6}{*}{9} & & .90 & & .98 & & .99 & & .93 \\
\hline & & \multicolumn{5}{|c|}{ correlation of residuals for items 5 and 6} & & \\
\hline & .50 & & .63 & & .50 & & .51 & \\
\hline & & \multicolumn{5}{|c|}{ correlation of avoidance and anxiety factors } & & \\
\hline & \multirow{2}{*}{\multicolumn{2}{|c|}{.44}} & \multirow{2}{*}{\multicolumn{2}{|c|}{$\begin{array}{c}\text { father } \\
.37\end{array}$}} & \multirow{2}{*}{\multicolumn{2}{|c|}{$\begin{array}{c}\text { partner } \\
.29\end{array}$}} & \multicolumn{2}{|c|}{ best friend } \\
\hline & & & & & & & \multicolumn{2}{|c|}{.31} \\
\hline
\end{tabular}

Note. All parameters are statistically significant $(p<0.001) ; A V M=$ avoidance to mother, $\mathrm{AXM}=$ anxiety to mother, $\mathrm{AVF}=$ avoidance to father, $\mathrm{AXF}=$ anxiety to father, $\mathrm{AVP}=$ avoidance to romantic partner, $\mathrm{AXP}=$ anxiety to romantic partner, $\mathrm{AVBF}=$ avoidance to best friend, $A X B F=$ anxiety to best friend. In the table, there is four different models, separately for each attachment figure; thus, parameters of four different items are presented in each line.

The respecified models fit the data better than the original models. However, only the model for romantic partner fits the data well. The value of the RMSEA is too high, and chisquared tests are significant in all relational domains. However, this exact goodness-of-fit test is very sensitive to bigger samples and even trivial misspecification can produce a significant misfit (Ropovik, 2015). Simultaneously, there were no other theoretically justified options to improve the model, based on residual covariances, which would be theoretically justified at the same time. This respecified model was used in all the following analyses.

Respecified Model for All Relational Domains Together - Czech ECR-RS

A CFA for the complex model with all the 36 items and 8 freely correlated factors (both anxiety and avoidance factors for each attachment figure) is conducted on the subsample of participants answering only about their current romantic partner, $n=581$. The complex correlated-factor model fits the data well, see Table 6. Residual correlations of many items are evident from an inspection of the residual matrix across each attachment figure. The high correlation coefficient is primarily for items 5, 6, and 8, between answers to the mother domain and the father domain; next is item 4, between answers to the romantic partner domain and the best friend domain. These correlations could indicate the existence of a global factor, which is verified in the last part of Study 1 . Correlations between latent variables are presented in Table 3 , standardized parameter estimates are presented in Online Supplement (Table 1).

This model was also scalar invariant for men and women - responses to the ECRRS reveal 
Table 3 Correlations between latent factors in the complex respecified model - Czech sample

\begin{tabular}{|c|c|c|c|c|c|c|c|}
\hline & AVM & AXM & AVF & AXF & AVP & AVBF & AXBF \\
\hline \multicolumn{8}{|l|}{ AVM } \\
\hline AXM & $.51^{* * *}$ & & & & & & \\
\hline AVF & $.24^{* * *}$ & .04 & & & & & \\
\hline AXF & $.20^{* * *}$ & $.74^{* * *}$ & $.30^{* * *}$ & & & & \\
\hline AVP & $.26^{* * *}$ & $.13^{*}$ & $.16^{* *}$ & $.15^{* *}$ & & & \\
\hline AXP & $.19^{* * *}$ & $.37^{* * *}$ & $.14^{* *}$ & $.44^{* * *}$ & $.29^{* * *}$ & & \\
\hline AVBF & $.21^{* * *}$ & $.17^{* *}$ & $.21^{* * *}$ & $.19^{* * *}$ & $.22^{* * *}$ & $.11^{*}$ & \\
\hline $\mathrm{AXBF}$ & .05 & $.35^{* * *}$ & .09 & $.42^{* * *}$ & $.10^{*}$ & $.36^{* * *}$ & $.33^{* * *}$ \\
\hline \multicolumn{8}{|c|}{$\begin{array}{l}\text { Note } .{ }^{* * *} p<.001 ;{ }^{* *} p<.01 ;{ }^{*} p<.05 ; \mathrm{AVM}=\text { avoidance to mother, } \mathrm{AXM}=\text { anxiety to mother, } \\
\text { AVF = avoidance to father, AXF = anxiety to father, AVP = avoidance to romantic partner, } \\
\text { AXP = anxiety to romantic partner, AVBF = avoidance to best friend, AXBF = anxiety to best } \\
\text { friend. }\end{array}$} \\
\hline
\end{tabular}

that there is little variation in how men and women experience attachment avoidance and attachment anxiety. See Online Supplement (Table 2) for the result.

\section{Global Attachment Avoidance and Global Attachment Anxiety - Czech ECR-RS}

Two global factors are specified in the higher-order model-global attachment avoidance and global attachment anxiety. First-order variables for global attachment avoidance are represented by each specific factor of attachment avoidance (i.e., avoidance for mother, father). First-order variables for global attachment anxiety are represented by each specific factor of attachment anxiety. The parameter estimates are presented in Table 4.

For comparison, a bifactor model is suggested. The bifactor model is specified as follows: a) two global factors are added to the previously described respecified model and the variance of the global factors is fixed to 1 ; b) a correlation between specific attachment avoidance and specific attachment anxiety is allowed, but only within the relational domains (not across them) and a correlation between global factors is allowed; c) the orthogonality of specific and global factors is set. The parameter estimates are presented in Table 5.

The bifactor model is compared to the higher-order model, assuming the models are nested. The higher-order model and the bifactor model differ only in the setting of global factors. In this step, the hypothesis about the indirect/mediated influence (the higher-order model) and the direct influence of global factors (the bifactor model) are compared. The bifactor model is preferable for further analyses (see Table 6). It is necessary to highlight that it is impossible to determine whether these two models can be considered nested (Bentler \& Satorra, 2010) and therefore their comparison may be biased.

The results support the plausibility of global avoidance and anxiety factors as measured by the ECR-RS, and that the bifactor model is possibly preferred. For attachment avoidance, the results show that specific factors have a greater effect on item responses than the global factor. For global attachment anxiety, there is a greater effect of global anxiety 
Table 4 Standardized parameter estimations for higher-order model - Czech sample

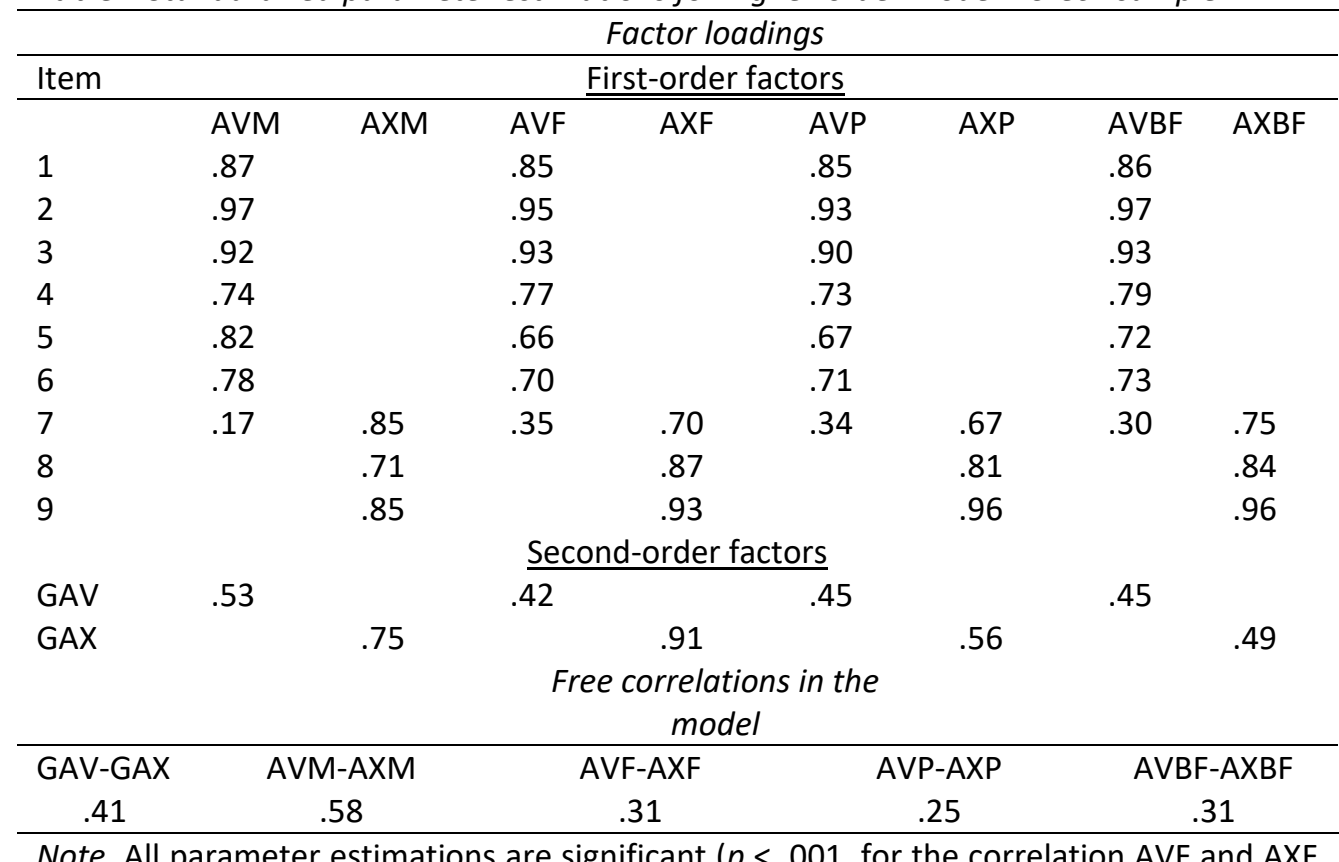

Note. All parameter estimations are significant $(p<.001$, for the correlation AVF and AXF $p<.01$ ); correlation of residuals: item 5 and 6: for AVM $r=.57$; AVF $r=.65$; AVP $r=.46$; AVBF $r=.47$; McDonald's coefficient omega is used to estimate the reliability of global factors reliability for global factors. The reliability analysis shows the following results: the second-order factors (global avoidance and global anxiety) explain the substantial proportion of variance of total score (for global avoidance: $\omega_{1}=.50$; global anxiety: $\omega_{1}=$ .76); the proportion of the manifest variable's variance is explained by high saturation of global factors after partializing the uniqueness from first-order factors (global avoidance: partial $\omega_{p}=.90$; global anxiety: partial $\left.\omega_{p}=.94\right) ; A V M=$ avoidance to mother, $A X M=$ anxiety to mother, $A V F=$ avoidance to father, $A X F=$ anxiety to father, $A V P=$ avoidance to romantic partner, $A X P=$ anxiety to romantic partner, $A V B F=$ avoidance to best friend, $A X B F=$ anxiety to best friend, GAV = global avoidance, $G A X=$ global anxiety. The model contained 36 items in total, 9 for each attachment figure. Thus, there are four different items in each row of the table.

on item responses in the mother and father domains, and there is an opposite trend for the partner and the best friend domains.

\section{Summary}

The results confirm the original 2-factor structure for each domain, notwithstanding the respecification of the model that had to be introduced to achieve acceptable fit for the observed data in each of the four relational domains. Measurement invariance analyses support invariance across gender. Global avoidance and global anxiety factors were supported with slight preference for the bifactor model, in which global attachment avoid- 


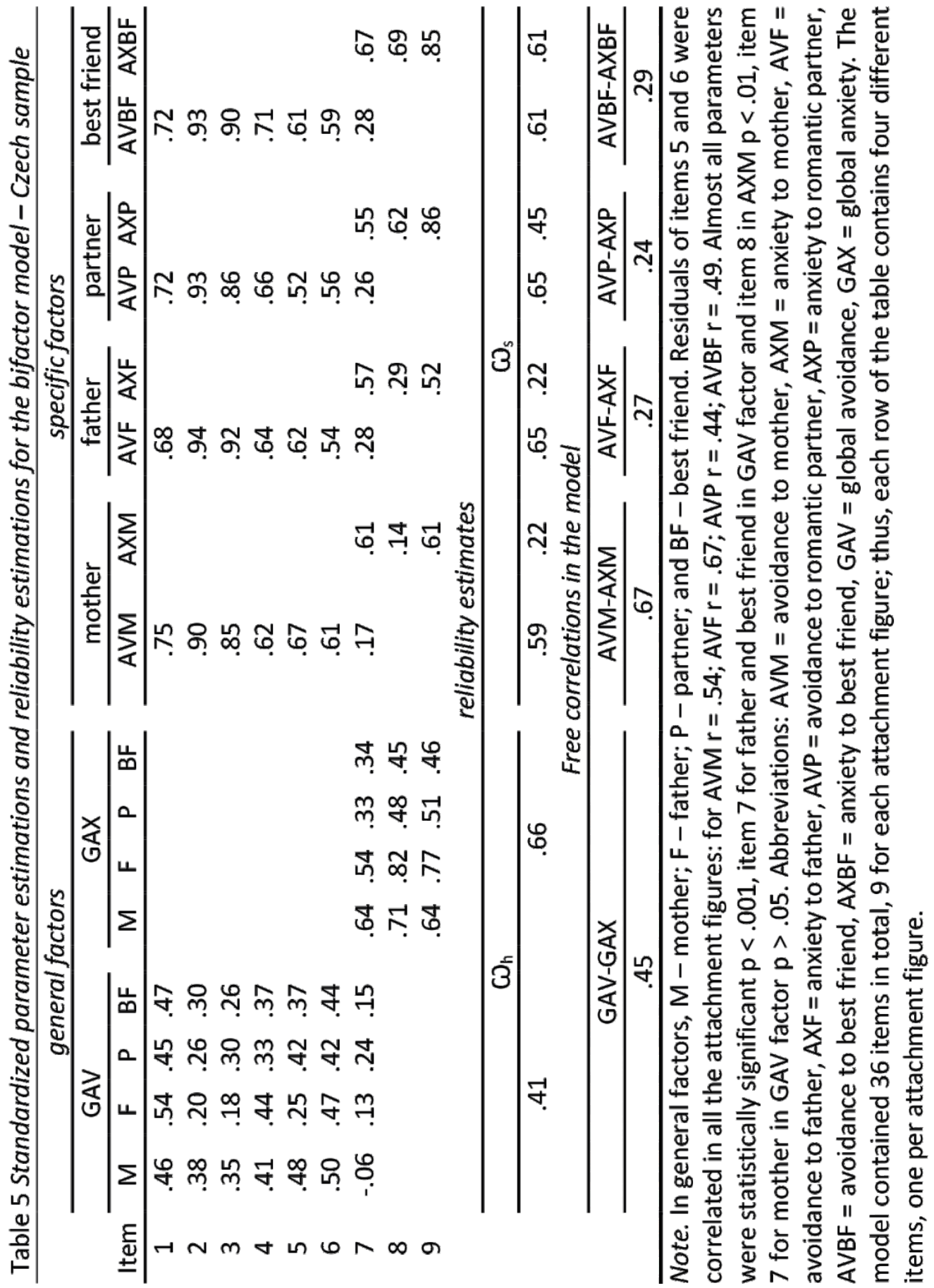


Table 6 Fit indices for the complex correlated-factor model, higher-order model and the bifactor model and their comparison-Czech sample

\begin{tabular}{lcccccc}
\hline Model & $\chi^{2}$ & $d f$ & $C F I$ & $T L I$ & $R M S E A\left[C l_{90 \%}\right]$ & SRMR \\
\hline $\begin{array}{l}\text { Correlated-factor } \\
\text { model }\end{array}$ & $1699.193^{* * *}$ & 559 & 0.966 & .961 & $\begin{array}{c}0.059 \\
{[0.056,0.063]}\end{array}$ & 0.066 \\
\hline $\begin{array}{l}\text { Higher-order } \\
\text { model }\end{array}$ & $1375.206^{* * *}$ & 574 & 0.980 & 0.978 & $\begin{array}{c}0.049 \\
{[0.046,0.052]}\end{array}$ & 0.066 \\
Bifactor model & $911.332^{* * *}$ & 543 & 0.991 & 0.989 & $\begin{array}{c}0.034 \\
{[0.030,0.038]}\end{array}$ & 0.049 \\
\hline
\end{tabular}

Note. ${ }^{* * *} p<.001$. Comparison of higher order and bifactor models: $\Delta \chi^{2}(31)=239.39$, $p<.001$.

ance and global attachment anxiety appear to directly relate to our experiences in relationships along with relationship-specific attachment avoidance and attachment anxiety influences.

\section{Study 2}

In Study 2, the same analyses are conducted as in Study 1 in order to replicate the English version of the ECR-RS.

\section{Method}

\section{Participants}

For Study 2, we used two cross-sectional datasets from the Open Science Framework website (OSF; https://osf.io/quk9a/), which were collected by Hudson et al. (2015). Analyses were performed on cases that met the following criteria: 1) self-reported age between 16 and 31 (the same as for the Czech sample) 2) English-speaking country 3) with current romantic partners and living parents 4) having indicated that they had not taken the survey before. Then we merged both samples into one dataset.
Data from 1,095 participants, who answered all relationship domains in the ECR-RS and met the criteria listed above, were used in this study. Their ages ranged from 16 to 30 years $(M=22.17, S D=3.74)$. The sample was composed primarily of women $(82 \%, n=$ 903). Most participants were from the United States $(n=770)$, with the remainder of the sample from Canada ( $n=175)$, the United Kingdom $(n=94)$, Australia $(n=32)$, Ireland $(n=11)$, New Zealand $(n=11)$, and Jamaica $(n=2)$. The average length of the romantic relationship was 4.77 months $(S D=3.75$, range: 0-26).

\section{Materials and Procedures}

In Study 2, the original version of The Experience in Close Relationships-Relationship Structures (Fraley et al., 2011) is used; for details, see Study 1. In our sample, the Cronbach's alpha reliability estimates for average scores were: "mother domain" $\alpha_{\text {avoid }}=0.91$, $\alpha_{\text {anx }}=0.88$; "father domain" $\alpha_{\text {avoid }}=0.92$, $\alpha_{\text {anx }}=0.89$; "partner domain" $\alpha_{\text {avoid }}=0.84$, $\alpha_{\text {anx }}=0.88$; "best friend domain" $\alpha_{\text {avoid }}=0.86$, $\alpha_{\mathrm{anx}}=0.86$. 


\section{Data Analysis}

First, the response categories were collapsed to the same 4-point scale, as in the Czech version, and the data were treated as ordinal. Second, the process of data analysis is exactly the same as in Study 1.

\section{Results}

\section{The Complex Model - English ECR-RS}

Because of the purpose of Study 3, we used the same respecified model as in Study 1. The complex correlated-factor model for the English ECR-RS fits the data well, see Table 5 , which cross-validated or model improvements. Patterns of factor correlations, standardized factor loadings and residual correlations were similar to Czech ECR-RS, see Online Supplement (Tables 4 and 5). Similarly to Study 1 , this model was also invariant across sex, see Online Supplement (Table 3).

Global Attachment Avoidance and Global Attachment Anxiety - English ECR-RS

We specified higher-order and bifactor models exactly in the same way as in Study 1. The pattern of their parameters was very similar to the Czech
ECR-RS in Study 1 (for details, see Online Supplement, Tables 6 and 7). They fitted data very well, which cross-validated our specification of both models with general factors on different sample; for fit indices, see Table 7.

The bifactor model is again compared to the higher-order model, assuming the models are nested. In this step, the hypothesis about the indirect/mediated influence (the higher-order model) and the direct influence of global factors (the bifactor model) are compared for the English ECR-RS. Fit indices for both models are presented in Table 7. Again, the bifactor model appears to fit the data better.

The results again suggest that specific factors have greater effect on items than the global factor in attachment avoidance. Specific attachment anxiety has higher effect on items regarding the partner and the best friend. However, global and specific attachment anxiety relates to items almost to the same degree for items regarding the father, but specific attachment loadings are slightly higher. Global attachment appears to have higher effect on items regarding the mother.

\section{Summary}

The findings cross-validate our previous conclusions from the analysis on the Czech sam-

Table 7 Fit indices for the higher-order model and the bifactor model and its mutual comparison - English sample

\begin{tabular}{lcccccc}
\hline Model & $\chi^{2}$ & $d f$ & $C F I$ & TLI & RMSEA [Cl 90\% $\left._{0}\right]$ & SRMR \\
\hline Correlated-factor model & $2223.382^{* * *}$ & 559 & 0.980 & 0.977 & $\begin{array}{c}0.052 \\
{[0.050,0.054]}\end{array}$ & 0.049 \\
\hline Higher-order model & $1806.943^{* * *}$ & 574 & 0.985 & 0.983 & $\begin{array}{c}0.044 \\
{[0.042,0.047]}\end{array}$ & 0.053 \\
Bifactor model & $1458.278^{* * *}$ & 543 & 0.989 & 0.989 & $\begin{array}{c}0.039 \\
{[0.037,0.042]}\end{array}$ & 0.043 \\
\hline
\end{tabular}

Note. ${ }^{* * *} p<.001$; Model comparison of higher order and bifactor model: $\left(\Delta \chi^{2}(31)=172.45\right.$, $p<.001)$. 
ple in Study 1. A two-factor structure of ECRRS for each attachment figure was supported. The plausibility of general attachment factors was supported too, with slight preference of the bifactor structure as in the Czech sample.

\section{Study 3}

The purpose of Study 3 was to compare the results of Study 1 and Study 2 by applying a multiple-group mean and covariance structures analysis (MACS; Little, 1997), which allows to test latent correlation invariance independently on the latent variances using models with a phantom variable with the same fit and degrees of freedom as the traditional CFA models.

\section{Method}

We used the same two samples as in previous studies. The analytical approach was basically the same as invariance testing described in Online Supplement, however MACS extends the traditional invariance tests. We used phantom variables to test the hypothesis about latent aspects of the construct, i.e., equality of the latent correlations, and simultaneously control the measurement invariance of measurement indicators explicitly in one model (Little, 1997). In this model, the phantom variable is a second-order variable and it predicts all the variance of its associated first-order variable, e.g., avoidance to mother or global avoidance. To implement phantom variables, we fixed the residual variances of first-order factors at 0 , variances of phantom variables were fixed at 1 , and loadings of phantom variables are freely estimated for both groups. These loadings can be interpreted as the standard deviations of the latent traits.

Then, the first three steps of MACS are identical to the measurement invariance testing described in Study 1 (configural, metric, and scalar invariance). In the fourth step, thresholds and loadings are still constrained across groups, but intercepts and latent variable means are set to zero for both groups, and latent variable variances are freely estimated (hypothesis that latent means do not differ across groups). The fifth step is divided into two parts, both are compared to step four: 5a) thresholds, loadings, and latent variable variances are constrained across groups, intercepts and latent variable means are set to zero for both groups (hypothesis, that latent variances do not differ across groups); 5b) threshold, loadings, latent variable variances, and latent variable correlations are constrained across groups, intercepts and latent variable means are set to zero for both groups (hypothesis: latent correlations do not differ across groups).

\section{Results}

The results of multiple group mean and covariance structures analysis (MACS) are presented in Table 8. The results support measurement invariance for the Czech and the English version of ECR-RS - despite the $\chi^{2}$ tests being significant, the difference fit indices were below reasonable cut scores (Chen, 2007; Sass, Schmitt, \& Marsh, 2014). According to Little (1997), we can meaningfully test the hypotheses about the differences/similarities of latent constructs in each group.

We added two models, one with constrained latent variable variances (5a) and one with constrained latent variable correlations (5b). According to our results (changes in fit indices are negligible), we can conclude that attachment avoidance and attachment anxiety do not demonstrate different relations either on the specific level or the global level across the Czech sample and the sample of English-speaking countries. 
Table 8 The results of multiple-group mean and covariance structure analysis

\begin{tabular}{|c|c|c|c|c|c|c|c|}
\hline & $\chi^{2}$ & $d f$ & $\Delta \chi^{2}$ & $\Delta d f$ & $T L I$ & RMSEA [Cl $\left.{ }_{90 \%}\right]$ & SRMR \\
\hline 1 & $2296.89^{* * *}$ & 1086 & & & 0.989 & $\begin{array}{c}0.036 \\
{[0.034,0.039]}\end{array}$ & 0.046 \\
\hline 2 & $2950.14^{* * *}$ & 1190 & $310.54^{* * *}$ & 104 & 0.985 & $\begin{array}{c}0.042 \\
{[0.04,0.044]}\end{array}$ & 0.053 \\
\hline 3 & $3331.31^{* * *}$ & 1252 & $383.90^{* * *}$ & 62 & 0.983 & $\begin{array}{c}0.045 \\
{[0.043,0.046]}\end{array}$ & 0.054 \\
\hline 4 & $3900.48^{* * *}$ & 1262 & $142.78^{* * *}$ & 10 & 0.979 & $\begin{array}{c}0.05 \\
{[0.048,0.052]}\end{array}$ & 0.053 \\
\hline $5 a$ & $3923.21^{* * *}$ & 1272 & $22.89^{*}$ & 5 & 0.979 & $\begin{array}{c}0.05 \\
{[0.048,0.052]}\end{array}$ & 0.054 \\
\hline $5 b$ & $4014.4^{* * *}$ & 1267 & $20.47^{*}$ & 10 & 0.978 & $\begin{array}{c}0.051 \\
{[0.049,0.053]}\end{array}$ & 0.057 \\
\hline
\end{tabular}

Note. ${ }^{* * *} p<.001,{ }^{*} p<.05 ; 1$ - configural model, no identification constraints; 2 - thresholds and loadings are constrained across groups, intercepts are freed for the second group, latent variable variances are free estimated and latent variable means are set to zero; 3 thresholds, loadings, and intercepts are constrained across groups, latent variables variance for both group and latent variable means for the second group are free estimated; 4 thresholds and loadings are constrained across groups, intercepts and latent variable means are set to zero for both groups, latent variable variances are free estimated; $5 \mathrm{a}$ - thresholds, loadings, and latent variable variances are constrained across groups, intercepts and latent variable means are set to zero for both groups; $5 b$ - thresholds, loadings, latent variable variances, and latent variable correlations are constrained across groups, intercepts and latent variable means are set to zero for both groups.

\section{Summary}

We provided the evidence of the measurement and structural invariance of the bifactor model using Czech and English version of ECRRS. The result supported possible intercultural generalizability of the bifactor structure of attachment in adulthood, at least between the international sample (English version of questionnaire) and the Czech sample (Czech version of the method).

\section{Discussion}

Results of the present studies provide additional evidence of the factor structure of the ECR-RS. The results confirm the original 2-factor structure for each domain, notwithstanding the respecification of the model that had to be introduced to achieve acceptable fit for the observed data in each of the four relational domains. Measurement invariance analyses support invariance across gender in both the Czech and the original English versions of the ECR-RS. Our next important aim was to examine the possibility to extract global attachment (global avoidance and global anxiety). The bifactor model was the best-fitting model, so global attachment avoidance and global attachment anxiety appear to directly relate to our experiences in relationships along with relationship-specific attachment avoidance and attachment anxiety. 
Global and specific attachment are orthogonal variables. We replicated this result (Study 2 ) on the original version of the ECR-RS, and we provided possible cross-cultural evidence of the measurement and structural invariance of this finding (Study 3).

\section{The Factor Structure of the ECR-RS}

Published studies support the original 2-factor structure of various language adaptations of the ECR-RS (i.e., Moreira et al., 2015) and various ECR measures (i.e., Sibley \& Liu, 2004). Our results do not confirm the factor structure of the ECR-RS completely - the residual correlation between items 5 and 6 and the cross-loading of item 7 had to be allowed. The non-zero residual correlation between items 5 and 6 could be due to their negative wording (the preceding four items of the attachment avoidance scale are positively worded) or with item order (i.e., Roszkowski \& Soven, 2010). A model that could control positive and negative formulation could not be identified because of the low number of negative worded indicators. Another possible explanation is that the content meaning of these items is very similar. The same issue was reported by Moreira et al. (2015) with the Portuguese version of the ECR-RS.

The necessity of allowing the cross loading of item 7 could be a consequence of the item's position in the measure. Item 7 is the first item of the attachment anxiety scale after six avoidance items - "setting" from previous six items could influence the answer to item 7. Unfortunately, we were not able to verify this hypothesis as we did not manipulate the order of the items in the Czech sample, and also the original cross-sectional dataset (Hudson et al., 2015) used the stable item order. On the other hand, it is probable that the concern that someone "does not really care for us" would be significantly related to discom- fort in depending on others and in opening up to them.

It is also necessary to highlight that the respecification of models was also based on values of modification indices. According to Kline (2016), the values of modification indices could be biased by misspecification of the model (original model) so our respecified model may not replicate on a different sample. Additionally, the respecified models for the individual attachment figures do not fit the data very well, even after the respecification (except the model for romantic partner). The models described the correlation matrix well (based on SRMR values), but high RMSEA values signaled the necessity to free other parameters. Despite this, we did not identify any further theoretically meaningful modifications of the model.

The complex model fits the data well. It is possible that the complex model reflects the response process better (i.e., answers to mother could influence answers to father and so on). In the complex model, intercorrelations of attachment anxiety and attachment avoidance were allowed among different attachment figures (i.e., the correlation between attachment avoidance to mother and attachment avoidance to partner and so on), which improved fit statistics. Almost all factors correlated significantly, a strong association was supported especially for attachment anxiety to mother and father. These results are partially consistent with suggested global models according to the traditional theory about the stronger association between attachment to people from the same social category (i.e., Collins \& Read, 1994; Overall et al., 2003). Notwithstanding, the difference in the strength of the associations between attachment avoidance and attachment anxiety across different relational contexts are also consistent with the connectionist perspective, which highlights the similarity of psycho- 
logical traits over belonging to the same social category (Fraley, 2007). In line with the results of Moreira et al. (2015), we found middle to high association between all measures of attachment anxiety, whereas for attachment avoidance we did not find such strong associations. This pattern was consistent in both samples and is discussed below with relation to the hierarchical and bifactor models.

\section{Factor Invariance Across Gender}

To the best of our knowledge, this is the first study to evaluate measurement invariance of the ECR-RS across gender. Our results strongly support measurement invariance between males and females in both samples. The ECRRS items measure the same factors (attachment avoidance and attachment anxiety) in an identical way for both women and men. This finding is consistent with the findings of a measurement invariance analysis across gender in ECR-R (Favez et al., 2016).

\section{Global Attachment Avoidance and Global Attachment Anxiety}

Our next focus was to assess the plausibility of global attachment anxiety and global attachment avoidance factors in data comprising answers related to four attachment figures. We also tried to compare the relationship between possible global factors (global attachment) and specific factors (attachment to a concrete person) using the hierarchical structural model and the bifactor model. Our results strongly support the existence of global and specific attachment representation consistent with previous findings (i.e., Fraley, 2002; Collins \& Read, 1994). To the best of our knowledge, we are the first to implement a bifactor model of ECR-RS for the comparison of the relationship between global and specific attachment representation.
The bifactor model fits the data slightly better than the hierarchical model. The model assumes global factors (global attachment avoidance and global attachment anxiety) are orthogonal to specific factors (i.e., attachment avoidance/anxiety to mother, father). For example, attachment avoidance items for mother are loaded on both attachment avoidance to mother and global attachment avoidance, which loads on all other attachment avoidance items (attachment avoidance to father, partner, and best friend). The same principle is applied to other attachment figures for both attachment avoidance and attachment anxiety.

A comparison of the hierarchical model and the bifactor model suggests new information about the role and significance of global factors in attachment theory. The hierarchical model in the context of attachment presumed that global attachment avoidance influences how we perceive avoidance in the relationships with particular attachment figures. Similarly, the hierarchical model presumed that global attachment anxiety influences perceived anxiety in particular relationships. These assumptions were not fully supported in our study, considering the bifactor model fits the data better. Our data supports the hypothesis that global attachment avoidance and global attachment anxiety directly influence our experience, which is also influenced by specific attachment only to one particular attachment figure. This specific attachment is independent of global attachment. Global attachment avoidance and anxiety are not manifesting "through" a specific relationship (i.e. relationship with mother, father), but probably directly influence how we experience the relationship in general. Our experiencing of relationships is also influenced by the specifics of the particular relationship.

The bifactor model allows us to compare the influence of global and specific fac- 
tors on the responses to items. For attachment avoidance, specific factor loadings (attachment avoidance to mother, avoidance to father) are stronger in comparison to global factor loadings. It is necessary to highlight that global attachment avoidance showed low reliability. The four considered attachment figures probably do not represent the network of attachment sufficiently in adulthood. Other potential attachment figures could be siblings, other friends, colleagues. We assume that the involvement of more possible attachment figures could increase the reliability estimation for global attachment avoidance.

For attachment anxiety, global factor loadings are generally stronger in comparison to specific factor loadings primarily for answers corresponding to mother. For answers about partner and best friend, we observed stronger specific factor loadings. It is possible that global attachment anxiety is a more dispositional characteristic. From the developmental perspective, the relationships to mother and possibly father may have a greater influence on the development of dispositional attachment anxiety, which could be reflected as the global factor by responses to attachment anxiety items for mother and father. This explanation is consistent with the "prototype hypothesis", which suggests a stronger influence of early attachment representation on attachment behavior over a lifespan (Fraley, 2002). This pattern was not observed in attachment avoidance. We assume that attachment avoidance may be more influenced by the current situation in the relationship compared to attachment anxiety relatively.

It is necessary to highlight that this study operated with the two-factor model - two global factors (global attachment avoidance and global attachment anxiety). Previous studies on global attachment did not differentiate between global attachment avoidance and global attachment anxiety (i.e., Fraley, 2007; Overall et al., 2008), which was not supported by our data as the global factor correlations were medium in all models. The different influences of both factors are a new finding, and further research is needed to replicate our results. It is also important for future studies to examine the construct validity of global factors and focus on the association of global and specific factors with other variables.

In Study 3, the results supported the same relations for attachment avoidance and attachment anxiety both on the specific and global levels across the Czech sample and the sample of English-speaking countries. Our results supported the hypothesis about generalizability of the latent aspects of attachment avoidance and attachment anxiety across cultures. Our results are consistent with the study of Wei, Russell, Mallinckrodt, and Zakalik (2004), which support measurement invariance across different culture groups using the ECR (the previous of the ECR-RS). Notwithstanding, we compared single Czech national sample (Czech version of ECRRS) with an international Englishspeaking sample, which limited such a conclusion. Further research is necessary to investigate the idea of possible intercultural generalizability.

\section{Limitations}

We need to note that the results of our study can be applied only to young adults. The character of the relationship with a romantic partner can vary considerably in comparison to an older group. The results of our analysis might also be influenced by changes in attachment hierarchy during this age phase (Umemura, Lacinová, Macek, \& Kunnen, 2016). In addition, high variability in the length of romantic relationship was observed. Further studies should verify our analysis in a sample with a higher age range.

Another notable limitation is the exclusion of participants who did not respond to all re- 
lationship domains in the ECR-RS. Responding could have been perceived as lengthy, and the same repeated questions from different relationship domains decrease the motivation to complete the questionnaire. Additionally, respondents may not have responded to a particular relational domain because of a negative perception of the relationship or the absence of an attachment figure in one or more of the four offered relational domains. Further studies could consider possible reasons for not responding or not completing questions related to a concrete person, i.e., through mixed-methods design.

Other limitations are the cross-sectional design and the small sample size of subgroups (i.e., men). Another problem is in item ordering. We used the original version of ECRRS (Fraley et al., 2011) with a fixed item order. Unfortunately, in this version, first four items from the same factor are reversed scored. This could introduce response bias and cause a two-factor structure (at least to some degree) by the so-called "method effect". This has to be investigated in future studies; we strongly recommend using random item order. We also need to highlight that the comparison of the bifactor and the hierarchical models could be biased by statistical shortcomings, as both models are not necessarily nested (see Satorra \& Bentler, 2010, for details). The other problem with a model fit comparison is the so-called proportionality constraint of the hierarchical model in comparison to the bifactor model (see e.g., Gignac, 2016, for details). The hierarchical model can be viewed as the bifactor model with some additional constraints, which usually decrease model fit. If this proportionality is violated, the bifactor model is almost always favored (Cucina \& Byle, 2017). Also, recent simulation studies (e.g, Morgan, Hodge, Wells, \& Watkins, 2015; Murray \& Johnson, 2013) showed that bifactor models performed better than hierarchical ones, even if the population "true" model is hierarchical. Some authors, therefore, were concerned about the validity of bifactor models for several reasons (see Bonifay, Lane, \& Reise, 2016, or Reise, Kim, Mansolf, \& Widaman, 2016, for more examples). Though the mentioned simulation studies usually worked with simple bifactor models with one general factor and several nested specific factors, these concerns apply also to our more complex model. Thus, structural equation modeling cannot provide conclusive evidence to prefer bifactor or hierarchical structure of attachment. On the other hand, we supported at least the hypothesis that both general anxiety and avoidance factor can be measured using methods involving attachment to specific figures (e.g., mother, father) as in ECRRS.

\section{Conclusion}

Using appropriate analysis, the ECR-RS is a valuable method for measuring different aspects of attachment and it allows us to evaluate the complexity of attachment. This paper provided empirical support for the existence of two global attachment dimensions (global attachment anxiety and global attachment avoidance) that relates to our experience in relationships together with specific attachment avoidance and specific attachment anxiety. The degree of influence of global or specific aspects of attachment depends on the particular type of relationship (i.e., mother, father, partner, best friend). Attachment avoidance and attachment anxiety do not demonstrate different relations on both specific and global levels across the Czech sample and the sample of English-speaking countries. Our results possibly support the idea of the generalizability of the latent aspects of attachment avoidance and attachment anxiety across cultures and the cross-cultural validity of ECRRS scale, but it is necessary to conduct further research to reach this conclusion. 


\section{Acknowledgement}

This work was supported by the grant project of the Czech Science Foundation under grant number GA17-09797S.

\author{
Authors' ORCID \\ Aneta Siroňová \\ https://orcid.org/0000-0001-6177-688X \\ Hynek Cígler \\ https://orcid.org/0000-0001-9959-6227 \\ Stanislav Ježek \\ https://orcid.org/0000-0003-3848-1578 \\ Lenka Lacinová \\ https://orcid.org/0000-0002-7417-3073
}

\section{References}

Ainsworth, M. S., Blehar, M. C., Waters, E., \& Wall, S. (1978). Patterns of attachment: A psychological study of the strange situation. Oxford, England: Lawrence Erlbaum.

Baldwin, M. W., Keelan, J. P. R., Fehr, B., Enns, V., \& Koh-Rangarajoo, E. (1996). Social cognitive conceptualization of attachment working models: Availability and accessibility effects. Journal of Personality and Social Psychology, 71, 94-104. https://doi.org/10.1037/0022-3514.71.1.94

Biernat, M. (2003). Toward a broader view of social stereotyping. The American Psychologist, 58(12), 10191027. https://doi.org/10.1037/0003-066X.58.12.1019

Bonifay, W., Lane, S. P., \& Reise, S. P. (2017). Three concerns with applying a bifactor model as a structure of psychopathology. Clinical Psychological Science, 5(1), 184-186. https://doi. org/10.1177/2167702616657069

Brennan, K. A., Clark, C. L., \& Shaver, P. R. (1998). Self-report measurement of adult attachment: An integrative overview. In J. A. Simpson \& W. S. Rholes (Eds.), Attachment theory and close relationships (pp. 46-76). New York, NY, US: Guilford Press.

Cai, L. (2010). A two-tier full-information item factor analysis model with applications. Psychometrika 75(4), 581-612. https://doi.org/10.1007/s11336-010$\underline{9178-0}$
Cameron, J. J., Finnegan, H., \& Morry, M. M. (2012). Orthogonal dreams in an oblique world: A meta-analysis of the association between attachment anxiety and avoidance. Journal of Research in Personality, 46(5), 472-476. https:// doi.org/10.1016/i.jrp.2012.05.001

Collins, N. L., \& Read, S. J. (1994). Cognitive representations of adult attachment: The structure and function of working models. In K. Bartholomew \& D. Perlman (Eds.), Advances in personal relationships: Vol. 5. Attachment processes in adulthood (pp. 53-90). London: Jessica-Kingsley.

Crowell, J. A., Fraley, R. C., Roisman, G. I. (2016). Measurement of individual differences in adult attachment. In J. Cassidy \& P. R. Shaver (Eds.), Handbook of attachment: Theory, research, and clinical applications (3rd ed., pp. 598-638). New York, NY: Guilford Press.

Cucina, J., Byle, K. (2017). The bifactor model fits better than the higher-order model in more than $90 \%$ of comparisons for mental abilities test batteries. Journal of Intelligence, 5(27), 1-21. https://doi.org/10.3390/iintelligence5030027

Del Giudice, M. (2011). Sex differences in romantic attachment: A meta-analysis. Personality \& Social Psychology Bulletin, 37(2), 193-214. https:// doi.org/10.1177/0146167210392789

Epskamp, S. (2014). semPlot: Path diagrams and visual analysis of various SEM packages' output. $\mathrm{R}$ package version 1.0.1. Retrieved from http:// cran.r-project.org/package $=$ semPlot

Favez, N., Tissot, H., Ghisletta, P., Golay, P., \& Cairo Notari, S. (2016). Validation of the French version of the Experiences in Close Relationships-Revised (ECR-R) adult romantic attachment questionnaire. Swiss Journal of Psychology, 75(3), 113-121. https://doi.org/10.1024/1421 $\underline{0185 / a 000177}$

Feddern Donbaek, D., \& Elklit, A. (2014). A validation of the Experiences in Close Relationships-Relationship Structures scale (ECR-RS) in adolescents. Attachment \& Human Development, 16(1), 58-76. https://doi.org/10.1080/14 $\underline{616734.2013 .850103}$

Fraley, R. C. (2002). Attachment stability from infancy to adulthood: Meta-analysis and dynamic modeling of developmental mechanisms. Personality \& Social Psychology Review, 6(2), 
123-151. https://doi.org/10.1207/S15327957PSPR0602 03

Fraley, R. C. (2007). A connectionist approach to the organization and continuity of working models of attachment. Journal of Personality, 75(6), 11571180. https://doi.org/10.1111/j.1467-6494.2007. 00471.x

Fraley, R. C., Heffernan, M. E., Vicary, A. M., \& Brumbaugh, C. C. (2011). The Experiences in Close Relationships-Relationship Structures questionnaire: A method for assessing attachment orientations across relationships. Psychological Assessment, 23(3), 615-625. https://doi. org/10.1037/a0022898

Fraley, R. C., Hudson, N. W., Heffernan, M. E., \& Segal, N. (2015). Are adult attachment styles categorical or dimensional? A taxometric analysis of general and relationship-specific attachment orientations. Journal of Personality and Social Psychology, 109(2), 354-368. https://doi. org/10.1037/pspp0000027

Fraley, R. C., \& Roisman, G. I. (2014). III. Categories or dimensions? A taxometric analysis of the Adult Attachment Interview. Monographs of the Society for Research in Child Development, 79(3), 36-50. https://doi.org/10.1111/mono.12112

Fraley, R. C., Waller, N. G., \& Brennan, K. A. (2000). An item response theory analysis of self-report measures of adult attachment. Journal of Personality and Social Psychology, 78(2), 350-365. https://doi. org/10.1037/0022-3514.78.2.350

Gignac, G. E. (2016). The higher-order model imposes a proportionality constraint: That is why the bifactor model tends to fit better. Intelligence, 55, 57-68. https://doi.org/10.1016/J.INTELL.2016.01.006

Gillath, O., Karantzas, G. C., \& Fraley, R. C. (2016). Adult attachment: A concise introduction to theory and research. Academic Press.

Griffin, D. W., \& Bartholomew, K. (1994). Models of the self and other: Fundamental dimensions underlying measures of adult attachment. Journal of Personality and Social Psychology, 67(3), 430-445. https://doi.org/10.1037/00223514.67.3.430

Hudson, N. W., Fraley, R. C., Chopik, W. J., \& Heffernan, M. E. (2015). Not all attachment relationships develop alike: Normative cross-sectional age trajectories in attachment to romantic partners, best friends, and parents. Journal of Research in Personality, 59, 44-55. https://doi. org/10.1016/j.jrp.2015.10.001

Chen, F. F. (2007). Sensitivity of goodness of fit indexes to lack of measurement invariance. Structural Equation Modeling: A Multidisciplinary Journal, 14(3), 464-504. https://doi. org/10.1080/10705510701301834

Kline, R. B. (2016). Principles and practice of structural equation modeling. London: The Guilford Press.

Klohnen, E. C., Weller, J. A., Luo, S., \& Choe, M. (2005). Organization and predictive power of general and relationship-specific attachment models: One for all, and all for one? Personality and Social Psychology Bulletin, 31(12), 1665-1682. https://doi.org/10.1177/0146167205278307

Little, T. D. (1997). Mean and Covariance Structures (MACS) analyses of cross-cultural data: Practical and theoretical issues. Multivariate Behavioral Research, 32(2), 53-76. https://doi. org/10.1207/s15327906mbr3201 3

Macek, P., Horská, E., Kotrlová, H., Kvitkovičová, L., Lacinová, L., \& Ježek, S. (2016). Paths to adulthood: Project and methods. Unpublished manuscript, Institute for Research on Children, Youth and Family, Faculty of Social Studies, Masaryk University, Brno, Czech Republic.

Moreira, H., Martins, T., Gouveia, M. J., \& Canavarro, M. C. (2015). Assessing adult attachment across different contexts: Validation of the Portuguese version of the Experiences in Close Relationships-Relationship Structures Questionnaire. Journal of Personality Assessment, 97(1), 22-30. https://doi.org/10.1080/00223891.2014.95037 $\underline{7}$

Morgan, G., Hodge, K., Wells, K., \& Watkins, M. (2015). Are fit indices biased in favor of bi-factor models in cognitive ability research?: A comparison of fit in correlated factors, higher-order, and bi-factor models via Monte Carlo simulations. Journal of Intelligence, 3(1), 2-20. https://doi. org/10.3390/iintelligence 3010002

Murray, A. L., \& Johnson, W. (2013). The limitations of model fit in comparing the bi-factor versus higher-order models of human cognitive ability structure. Intelligence 41(5), 407-422. https:// doi.org/10.1016/j.intell.2013.06.004

Overall, N. C., Fletcher, G. J. O., \& Friesen, M. D. (2003). Mapping the intimate relationship mind: 
Comparisons between three models of attachment representations. Personality and Social Psychology Bulletin, 29(12), 1479-1493. https:// doi.org/10.1177/0146167203251519

R Core Team (2016). R: A language and environment for statistical computing. R Foundation for Statistical Computing, Vienna, Austria. URL https://www.R-project.org.

Reise, S. P., Kim, D. S., Mansolf, M., \& Widaman, K. F. (2016). Is the bifactor model a better model or is it just better at modeling implausible responses? Application of iteratively reweighted least squares to the Rosenberg Self-Esteem Scale. Multivariate Behavioral Research 51(6), 818-838. https://doi.or $\mathrm{g} / 10.1080 / 00273171.2016 .1243461$

Ropovik, I. (2015). A cautionary note on testing latent variable models. Frontiers in Psychology, 6, 1715. https://doi.org/10.3389/fpsyg.2015.01715

Rosseel, Y. (2012). lavaan: An R package for structural equation modeling. Journal of Statistical Software, 48(2), 1-36. Retrieved from http:// www.jstatsoft.org/v48/i02/

Roszkowski, M. J., \& Soven, M. (2010) Shifting gears: Consequences of including two negatively worded items in the middle of a positively worded questionnaire. Assessment \& Evaluation in Higher Education, 35(1), 113-130. https://doi. org $/ 10.1080 / 02602930802618344$

Sass, D. A., Schmitt, T. A., \& Marsh, H. (2014). Evaluating model fit with ordered categorical data within a measurement invariance framework: A comparison of estimators. Structural Equation Modeling: A Multidisciplinary Journal, 21(2), 167-180. https:// doi.org/10.1080/10705511.2014.882658
Satorra, A., \& Bentler, P. M. (2010). Ensuring positiveness of the scaled difference chi-square test statistic. Psychometrika. https://doi.org/10.1007/ s11336-009-9135-y

semTools Contributors. (2016). semTools: Useful tools for structural equation modeling. $\mathrm{R}$ package version 0.4-14. Retrieved from https:// CRAN.R-project.org/package=semTools

Sibley, C. G., \& Liu, J. H. (2004). Short-term temporal stability and factor structure of the revised experiences in close relationships (ECR-R) measure of adult attachment. Personality \& Individual Differences, 36(4), 969. https://doi. org/10.1016/S0191-8869(03)00165-X

Sibley, C. G., \& Overall, N. C. (2008). Modeling the hierarchical structure of attachment representations: A test of domain differentiation. Personality \& Individual Differences, 44(1), 238-249. https://doi.org/10.1016/i.paid.2007.08.003

Umemura, T., Lacinová, L., Macek, P., \& Kunnen, S. E. (2016). Longitudinal changes in emerging adults' attachment preferences for their mother, father, friends, and romantic partner: Focusing on start and end of romantic relationships. International Journal of Behavioral Development. Advance Online Publication. https://doi. org/10.1177/0165025416647545

Wei, M., Russell, D. W., Mallinckrodt, B., \& Zakalik, R. A. (2004). Cultural equivalence of adult attachment across four ethnic groups: Factor structure, structured means, and associations with negative mood. Journal of Counseling Psychology, 51(4), 408-417. https://doi.org/10.1037/0022$\underline{0167.51 .4 .408}$ 